\title{
COMPUTATIONAL IR SPECTROSCOPY FOR PAHS: FROM THE EARLY YEARS TO THE PRESENT STATUS
}

\author{
F. Pauzat ${ }^{1}$
}

\begin{abstract}
In the long story of interstellar PAHs, computations have played and are still playing a fundamental role in connection with experiments and observations. From the very first calculations of the IR spectra of small PAHs in the late eighties to the more recent ones, every aspect of the research linked to the PAH hypothesis has evolved dramatically: the size and the variety of the molecules considered, the techniques used, the precision of the astronomical observations ... The initial landscape has completely changed though the quest is still the same, that is to correlate the so-called UIR bands spectra ubiquitous in the ISM (Inter Stellar Medium) with a chemical family of molecules, the PAHs. An historical review of the 25 years of this quest is presented here, focusing on the computational part.
\end{abstract}

\section{Introduction}

Theoretical studies of chemical species and processes in extreme physical conditions are a clear need of the astrophysical community confronted with situations almost impossible to reproduce in laboratory experiments. Many questions arise that do not find experimental answers easily. However, some of them can be obtained from theoretical studies that are the alternative in a number of problems.

One of these questions, which has proved to be a formidable challenge in modern astrophysics, is the interpretation of the infrared emission bands of our galaxy between 2 and $15 \mu \mathrm{m}$. These bands, referred to as "Unidentified Infra Red (UIR) bands" in the literature, were first discovered by ground-based observations at the end of the seventies. Since then, their existence has been confirmed in a number of objects by observations using the IRAS, ISO and more recently Spitzer and AKARI space missions. This emission, characterized by a series of bands that are systematically found at $3.3,6.2,7.7,8.6,11.3$ and $12.7 \mu \mathrm{m}$, seems to be widely present in various environments of the interstellar medium (ISM). For interpreting

${ }^{1}$ UPMC Univ. Paris 06, UMR - CNRS 7616, LCT, 75005 Paris, France 
this emission, Léger \& Puget (1984), Allamandola et al. (1985) proposed a model based on the similarity between the spectra observed in space and those of neutral polycyclic aromatic hydrocarbons (PAH) obtained in the laboratory. In space, these molecules could become vibrationally excited by absorption of UV photons and then release their excess energy by means of infrared fluorescence (Duley \& Williams 1981). This is generally referred to as the PAH hypothesis.

Contrary to radio spectra whose interpretation relies on a perfect match between observations and measurements on a single molecule, the observed IR spectra correspond to families of molecules so that the details of the spectral match cannot be satisfactory; furthermore, the relative intensities of the different bands observed may vary significantly from an astronomical object to another. Various interpretations of these differences have been proposed, leading sometimes to contradictory conclusions such as severe dehydrogenation or negligible dehydrogenation of the interstellar PAHs. It has been said that PAHs could be either neutral or positively charged due to ionization by the radiation field and that they could be present in the form of negative ions within dense clouds (Wakelam \& Herbst 2008). Given such a diversity of hypotheses, the ideal strategy would have been to measure the IR emission of a selected number of representative species in the gas phase, at temperatures and densities reproducing the interstellar conditions in the laboratory. At that time, it was considered as a practically impossible task, and numerical simulation was the alternative. The main difficulties to be addressed were:

i) The size of molecules like PAHs, ranging from a few tenths to several hundreds of atoms, which was, when the study began, non tractable for most $a b$ initio quantum chemistry codes.

ii) The number of different types of molecules (ramified, substituted, ionized, hydrogenated, de-hydrogenated, metallic-complexes ...) to be treated at the same level of accuracy, implying a huge amount of computer time.

iii) The level of accuracy necessary to connect calculations with observations, which raises two more problems. The first one refers to a real theoretical challenge: one has to compare an emission spectrum (observed) to an absorption spectrum (computed). The second one is not relevant of pure quantum chemistry: the reference being the observed spectrum, i.e. a global spectrum emitted by a mixture of molecules from a known family but with an unknown composition, the comparison depends of the composition chosen and there is no theoretical criterion to help designing a unique solution.

The difficult quest for theoretical IR spectra usable by astrophysicists, has been extending from the last eighties to nowadays with successes as well as setbacks. The presentation will be split into three chronological parts. The first part retraces a "learning period", beginning in the late eighties, with methods whose reliability to efficiency ratio was still to be optimized for such molecules and variables. The options considered at the time are presented and discussed. It allowed to set up 
standard procedures and eventually led to some spectacular results. The second part deals with the following period which, for the computation side, turned into a systematic study of all possible PAHs. This mass production was undertaken to get databases available to the PAH community, in order to combine the efforts of theoreticians and experimentalists ( $c f$. Sect. 3.1). Indeed, mixing the results of calculations and of experiments turned out to be very fruitful. On top of those calculations, a large amount of work has been performed to produce synthetic spectra with variations of the type and size of the PAHs in order to compare directly with the observations and to deduce information about the population as a function of the environment (see for instance Cami et al. in this volume). The third part reports the main theoretical advances made during the last years, aiming at a much more precise insight into the spectroscopy of PAHs. The ongoing developments of new methods and the comparison of their results to astronomical spectra with fine structures of increasing complexity can be regarded as a real challenge. Going beyond the harmonic approximation and taking into account the distinction between emission and absorption spectra are the main points now addressed (see for example Basire et al. in this volume).

\section{First generation computations: HF vs. DFT electronic calculations}

The spectra observed between 2 and $50 \mu \mathrm{m}$ are the signatures of molecular vibrations. They are characterised by two quantities, namely, their frequencies and their intensities. The frequencies are the energetic signatures of the deformations of the molecular structure whereas the intensities show how the electronic density is distorted with the internal displacements of the nuclei. Together, they constitute a real fingerprint of the molecule, often used for identification in terrestrial laboratories.

Any non-linear molecule composed of $\mathrm{N}$ atoms can move along $3 \mathrm{~N}$ degrees of freedom, that can be separated into 3 translations and 3 rotations which do not imply any modification of the geometry, and $3 \mathrm{~N}-6$ vibrations (reduced to $3 \mathrm{~N}-5$ in the case of linear species) that involve periodic structural modifications with well defined frequencies.

Due to the quantum nature of the particles involved, quantum chemistry is most appropriate to describe the behavior of electrons and nuclei within a molecule. It is done by using mathematical functions capable of describing quantum phenomena from first principles and the universal constants of physics. Whatever the choices made, evaluation of wave functions or evaluation of electronic densities, all methods are based on solving the Schrödinger equation in which all the particles and all the interactions between them are taken into account. It is expressed as $H(r, R) \Psi(r, R)=E \Psi(r, R)$

where $H(r, R)$ is the complete Hamiltonian of the system depending on the electronic $r$ and nuclear $R$ coordinates. This operator is expressed as $H(r, R)=$ $T_{e}+T_{A}+V_{e A}+V_{e e}+V_{A A}$

where $T_{\mathrm{e}}$ and $T_{A}$ are the kinetic energy operators for the electrons and nuclei, $V_{e A}$ and $V_{\text {ee }}$ the operators describing the Coulomb attraction between electrons 
and nuclei and the repulsion between the electrons, $V_{A A}$ the repulsion between the nuclei.

Solving the Schrödinger equation is not a simple process since the energy to be calculated has contributions from various types of movements, motions of electrons, vibrations and rotations of the nuclear frame, all entangled in a subtle way. Rigorously, the Schrödinger equation can be solved exactly only for a two-electron atom. In all other cases, it is necessary to make approximations. Levering on the fact that nuclei are thousands times heavier than electrons, the first and most natural approximation is to uncouple the two types of motions (Born-Oppenheimer principle). Then, the calculations are carried out in the three following steps.

i) The first step is to determine the geometry of the molecule by minimizing its electronic energy as a function of geometrical parameters such as bond distances, bond angles, dihedral angles. It is a pure electronic calculation in which the wave function in the Schrödinger electronic equation, $\Psi(r, R)$, is a function depending explicitly of all the electronic variables (space and spin) and parametrically of the nuclei coordinates. $\Psi(r, R)$ is developed as a linear combination of suitable functions, known as basis sets. Different types of mathematical functions are currently used, as for example, atomic functions, plane waves or gaussian functions.

ii) The second step is to describe the movement of the nuclei in the potential previously calculated at fixed positions of the nuclei. Assuming that each vibration can be approximated by a harmonic oscillator, the so-called harmonic frequencies are obtained from the diagonalization of an effective force constant matrix built from the atomic masses and the second derivatives of the energy as a function of the displacements of the nuclei in the vicinity of their equilibrium positions. The eigenvalues lead to the harmonic vibrational frequencies whereas the eigenvectors provide the displacement vectors of the nuclei (normal coordinates). Going beyond the harmonic description would imply the mapping of a multidimensional energy potential allowing the calculation of higher order derivatives and inter-vibration coupling terms (see Sect. 4).

iii) The third step is the calculation of the vibrations intensities that are directly related to the first multipole moment that has a non vanishing derivative with respect to the normal coordinates previously obtained (Amos 1984; Helgaker et al. 1986).

\subsection{Independent electron model versus correlated electron model}

Back to the eighties, the methods of computational chemistry were already elaborated and could be very efficient but only for small sized molecules (3-4 atoms). Confronted to PAHs that are much larger molecules (several tens of atoms) and to the demand for an accurate determination of their IR frequencies and intensities that are wave function derivatives, quantum chemists could only use part of 
their codes, taking into account the computer possibilities of the time. Therefore, the first calculations were performed at the Hartree-Fock (HF) level; then came the Density Functional Theory (DFT) option, which is still the major theoretical scheme used today for the treatment of PAHs up to over a hundred of atoms.

In HF theory, electrons are assumed to be independent particles, each one moving in the average field of the others; there is no correlation between the electrons. Bringing correlation effects into the electronic wavefunction, i.e. taking into account that the movement of one electron is dependent on the instantaneous movement of the others, can be done in several ways. In post HF theories, correlation effects are introduced by mixing the ground state wavefunction with excited configurations. The simplest approach is known as MP2 (Moller Plesset 2nd order) in which only doubly excited configurations are considered in a perturbation treatment. In DFT, electrons are explicitely correlated through what is known as a correlation functional, which, by definition, makes it possible to limit the calculation to the ground state only. However, the exact correlation functional not being known, the DFT methods have to rely on approximate functionals. How the $\mathrm{HF}$ and DFT approaches are related is presented below.

In the HF theory, the energy as function of the density matrix $\mathrm{P}$, has the form ${ }^{1}$ : $E_{H F}=V+<h P>+1 / 2<P J(P)>-1 / 2<P K(P)>$

with:

$V$ : nuclear repulsion energy,

$<h P>$ : one-electron (kinetic + potential) energy

$1 / 2<P J(P)>$ : coulomb repulsion of the electrons

$-1 / 2<P K(P)>$ : exchange energy.

In the Kohn-Sham (KS) formulation of density functional theory, the energy has the form:

$E_{K S}=V+<h P>+1 / 2<P J(P)>+E_{X}[P]+E_{C}[P]$

with:

$E_{X}[P]$ : exchange functional

$E_{C}[P]$ : correlation functional.

Within the Kohn-Sham formulation, Hartree-Fock theory can be regarded as a special case of density functional theory, with:

$E_{X}[P]=-1 / 2<P K(P)>$ and $E_{C}=0$.

Although the inclusion of electron correlation in DFT can be seen as a qualitative leap in the description of electronic structures, one delicate issue remains when using DFT, namely, the choice of the exchange and correlation functionals. Some depend only of the local value of the density (LDA functionals), others depend not only of the local value of the density, but also of the derivative of this density (GGA functionals). Others, trying to take the best of the HF and DFT approaches use a combination of both (Hybrid functionals). This explains that the results can be rather different depending of the functional used. The most popular and widely

\footnotetext{
${ }^{1}$ The notation $<>$, known as the bra-ket Dirac formalism means that the quantity $\langle Q\rangle$ is obtained by integration over the wave function space.
} 
employed functional for the calculation of IR spectra is known under the acronym of B3LYP (Becke 1993; Lee et al. 1988). The form initially proposed by Becke for the exchange correlation functional depends on 3 parameters that are adjusted to fit molecular properties of a representative corpus, mostly of organic molecules, which explains the success of this approach in the PAH studies.

At that point, general textbooks (for example: Szabo \& Ostlund 1982; Lee \& Scuseria 1995; Koch \& Holthausen 2001) may help the interested reader to get a better insight into the original developments forming the basis of quantum chemistry as it stands today.

\subsection{Correcting the missing effects: Scaling procedures}

As mentioned before, all electronic methods are approximate when dealing with correlation, and at least part of the correlation effect is missing. Most of the time, evaluation of the IR frequencies is performed within the harmonic approximation. Thus, two independent effects, correlation and anharmonicity, have to be corrected. In such situations, the most efficient way to proceed is to adjust the raw values as a whole by calibrating on well known experimental data. Several procedures have been employed:

- a direct scaling of the frequencies using a unique scale factor for the entire spectrum or a specific scale factor by frequency range or frequency type,

- an indirect scaling through the force constant matrix, scaling only the diagonal terms (uniformly or not) or scaling also the non-diagonal terms.

The scaling factors depend essentially on the method and the basis set employed, and, in a lesser way, of the type of molecules considered (families). From the original work by Scott \& Radom (1996), a number of numerical experiments have been discussed in the literature (see: Yoshida et al. 2000; Merrick et al. 2007; Johnson et al. 2010; Bauschlicher \& Langhoff 1997, for the specific case of PAHs). One has to note that, contrary to frequencies, no scaling procedure is available for intensities which are linked to derivatives of observables.

\subsection{Successful applications}

The approximations detailed above should not mask the fact that an impressive amount of results has been obtained with these methods, allowing a real insight into the PAH spectroscopic features.

For example, a major question was how to justify the weakness of the $3.3 \mu \mathrm{m}$ feature observed, compared to the corresponding experimental value (known only for standard neutral PAHs at that time). Taking into account that this feature corresponds to the $\mathrm{CH}$ stretching vibration, the first idea that came in was to argue in favour of dehydrogenated PAHs, assuming the $\mathrm{CH}$ intensity to be proportional to the number of $\mathrm{CH}$ bonds. However, no spectrum of any dehydrogenated PAH was available and no data could support such an assumption, though it seemed a reasonable one. 


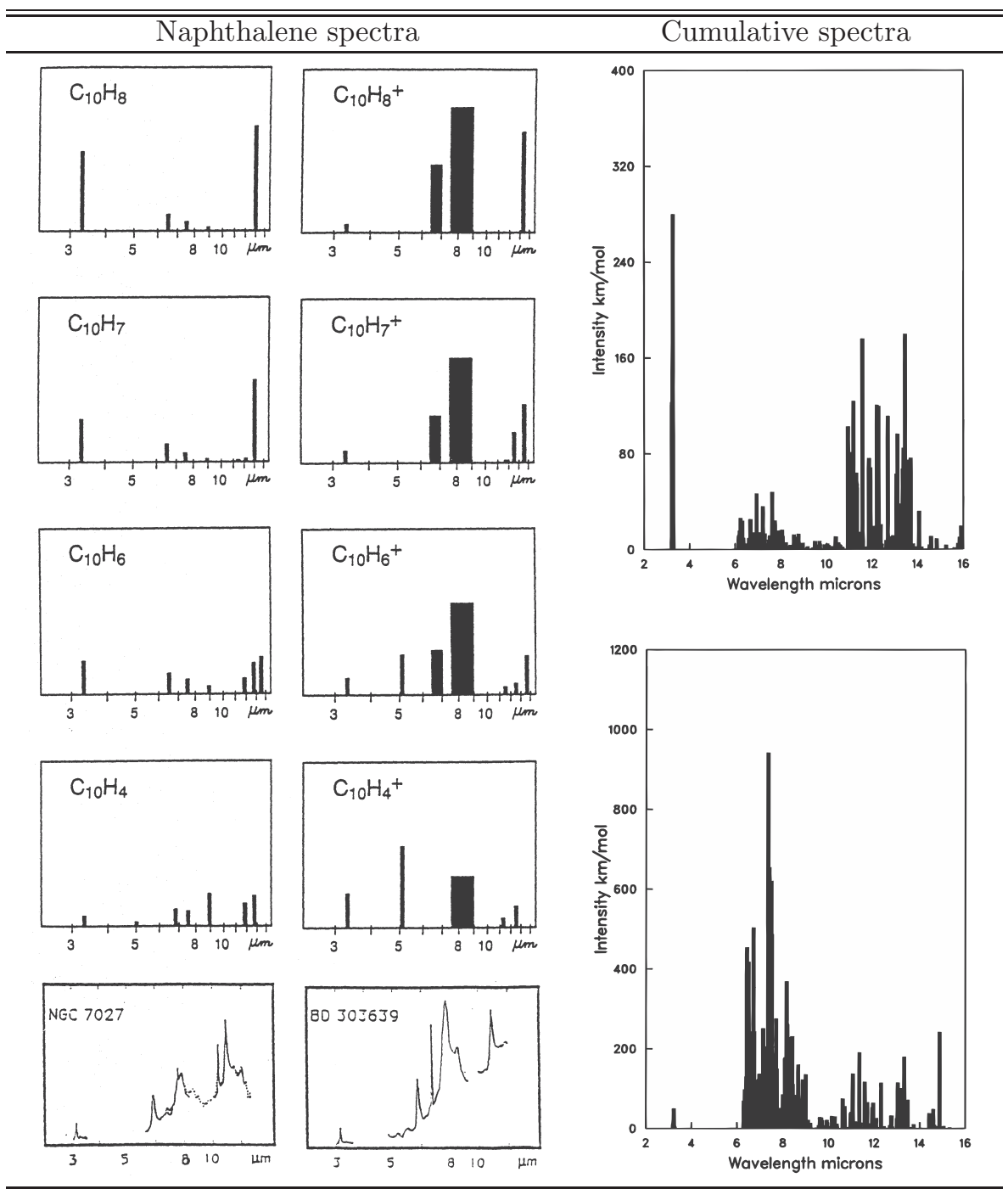

Fig. 1. Absorption spectra of neutral versus ionized PAHs. Left: effects of dehydrogenation and ionization in the naphthalene family $(\mathrm{HF} / 321 \mathrm{G}$ calculations with scaling factor specific of the type of vibration); the bands $F W H M$ are the averaged widths of the interstellar bands in the corresponding region of the spectra (adapted from Pauzat et al. 1995). Right: histogram representations of an equally weighted sum of the IR adsorption band intensities of 13 PAHs (B3LYP/4-31G with uniform scaling): neutrals (top) and cations (bottom) (adapted from Langhoff 1996). 
The first breakthrough came from quantum theory simulations showing the spectacular difference between neutrals and cations, in particular for the intensities of the $3.3 \mu \mathrm{m}$ feature. Suggested in 1988 by HF calculations for the smallest PAHs (Communication by Miller \& D. DeFrees, IAU Symp. 135, Interstellar Dust, Santa Clara), it was confirmed in 1992 jointly by computations (Pauzat et al. 1992) and experiments (Szczepanski et al. 1992). Later on, more extensive studies through HF (DeFrees et al. 1993) and DFT calculations (Langhoff 1996) as well as laboratory experiments emphasized this fact (see for example Oomens in this volume). The theoretical spectra reported in Figure 1 summarize the two aspects of that important result. First the compilation of the spectra of a series of 13 PAHs in their neutral and ionized forms (Langhoff 1996) illustrate the general collapse of the $3.3 \mu \mathrm{m}$ feature obtained by ionisation. Second, the case study of naphthalene (Pauzat et al. 1995) demonstrates that the collapse is effectively due to ionization of the PAH and not to some sort of dehydrogenation which would imply the same collapse of the $11.3 \mu \mathrm{m}$ feature and consequently could not account for the observed intensity ratios. This fact has been confirmed for larger species (Pauzat et al. 1997).

It is worth mentioning that the first generation computations have been precise and reliable enough to characterize the fluctuations in frequency positions and relative intensities connected to almost all the different families of PAHs suggested by the various interpretative models. Therefore, all sorts of PAH families have been calculated to support the interpretation of the observed IR spectra (see also Peeters in this volume). In addition to the large body of data obtained on neutral and singly ionized PAHs, more specific calculations were carried out on specifically targeted families. A non exhaustive list of examples is given below focusing on the physical effects, independently of the size of the PAH carriers. Among the effects considered one may cite:

- multiple ionizations (Ellinger et al. 1999; Bauschlicher \& Bakes 2000; Bakes et al. 2001; Malloci et al. 2007)

- negative ions (Langhoff 1996; Bakes et al. 2001; Bauschlicher et al. 2008, 2009)

- dehydrogenated PAHs (Pauzat et al. 1995, 1997)

- hydrogenated PAHs (Pauzat \& Ellinger 2001; Bauschlicher et al. 2001; Beegle et al. 2001; Ricks et al. 2009)

- substitutions by lateral chains and carbon replacements by heteroatoms (Langhoff et al. 1998; Pauzat et al. 1999; Hudgins et al. 2005)

- irregular structures with 4/5/7 membered rings (Bauschlicher et al. 1999; Pauzat \& Ellinger 2002)

- deuterated PAHs (Bauschlicher et al. 1997; Mulas et al. 2003; Peeters et al. 2004)

- metal complexation (Fe, Mg, Si) (Ellinger et al. 1999; Cassam 2002; Hudgins et al. 2005; Bauschlicher \& Ricca 2009; Joalland et al. 2010; Simon \& Joblin $2007,2010) \ldots$ 


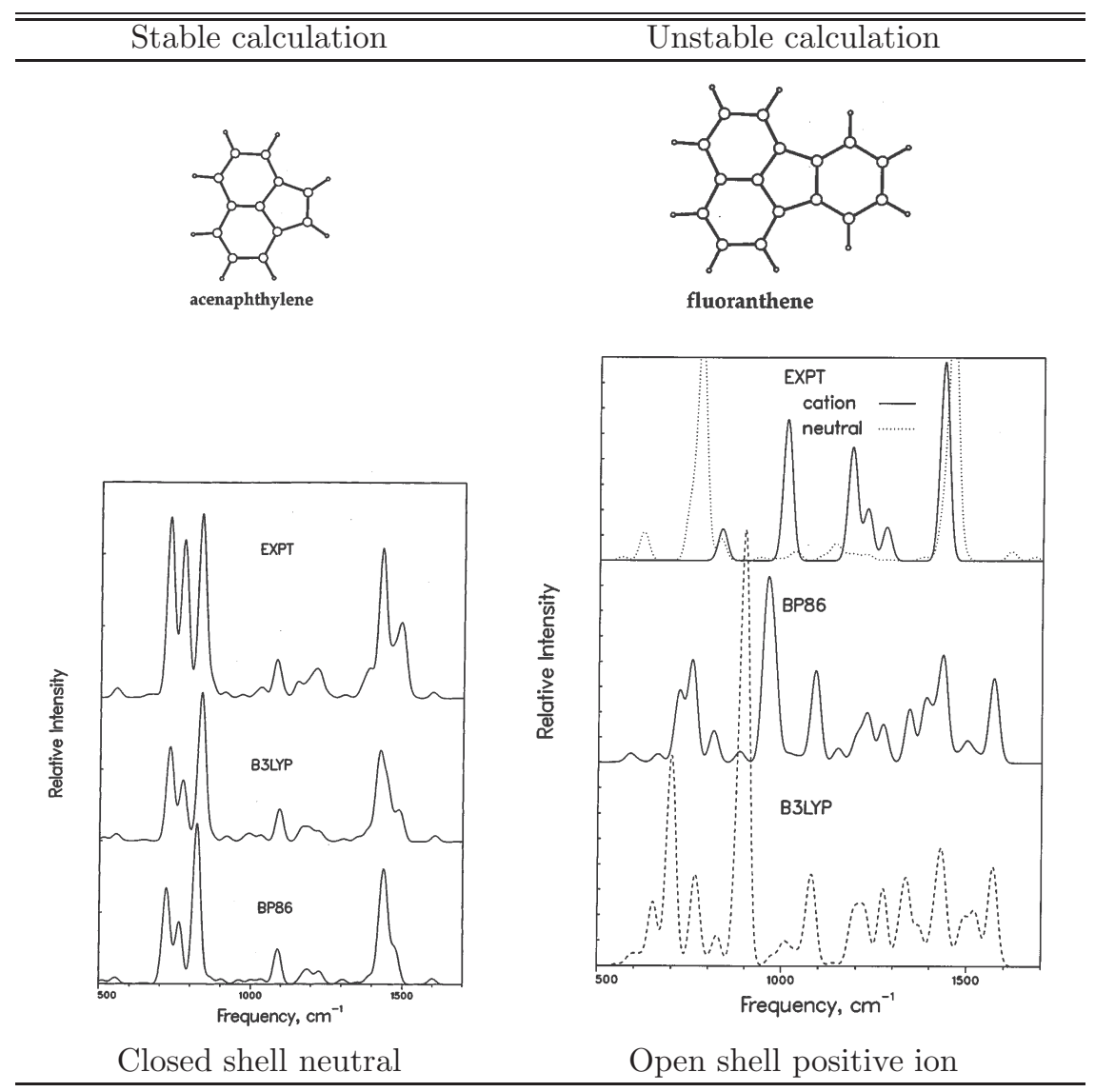

Fig. 2. Examples of successful (left) and unstable (right) calculations of IR spectra using BP86, B3LYP with the 4-31G basis set and $F W H M$ of $30 \mathrm{~cm}^{-1}$ (adapted from Bauschlicher et al. 1999).

\subsection{Limitations}

In spite of a number of successes and a satisfactory agreement with the experimental spectra when available (apart from some lack of precision for the intensities), some theoretical problems remain to be solved with electronic calculations. Instabilities, mostly due to symmetry breaking artefacts, may occur i.e. when the calculated electronic density has a lower symmetry than the nuclear frame. This happens when two states fall quite close in energy in an open shell structure. Practically, it generates abnormally large intensities as well as inaccurate positions of the bands, especially for in-plane asymmetric CC vibrations. Contrary to what is sometimes advanced, this is true with all methods used for PAHs but hopefully for a few molecules only. It is true for HF methods, especially when using ROHF for open-shells molecules (case of most cations), it is true for Moller Plesset 
Perturbation theory (MP2) which has been used sometimes as reference for correlation evaluation, and it is also true of DFT though less often. In this last case, it seems to depend on the choice of the functional. One of those cases, the fluoranthene cation, is discussed by Bauschlicher et al. (1999) considering B3LYP and BP86 DFT approaches (Fig. 2). However, the theoretically correct solution of symmetry breaking in open shells relying on high level multiconfigurational calculations (McLean et al. 1985) is still out of reach today for most PAHs because of the size of the computational problem.

\section{Second generation computations: Databases of PAHs IR spectra}

\subsection{PAHs databases}

Thanks to a quickly increasing power of the computers, systematic calculations of whole families of PAHs, dealing with larger and larger PAHs (Bauschlicher 2002; Pathak \& Rastogi 2007; Bauschlicher et al. 2008, 2009; Ricca et al. 2010), could be currently performed. The time had come for a PAH business, meaning large scale computations implying significant human and computer-time potentials. Such characteristics were available at NASA Ames Research Center. Moreover, experimental setups were available on the same site and cooperation between the two proved very successful. The same objective was also present in the European collaboration between the Cagliari Observatory and the CESR at Toulouse. Two databases are now currently on line:

The Cagliari database: astrochemistry.ca.astro.it/database/

For more details, refer to the review by Malloci et al. (2007):

The NASA database: www. astrochem.org/pahdb/

For more details, refer to the review by Bauschlicher et al. (2010) and see also Boersma et al. in this volume.

The exploitation of these on line databases of spectra is presented below.

\subsection{Modeling interstellar composite IR spectra}

Quantum Chemistry calculations give spectra of individual PAHs (free flyers) under a simple form: two numbers per vibration, the frequency and the corresponding absolute intensity; they provide also a vector array which is the normal coordinate describing the movement associated to the vibration in the reference frame of the molecular geometry. It is the analysis of the normal coordinates in terms of elementary displacements (stretching, bending...) that allows structural identification of the vibration. Starting from these data, one has to build a synthetic spectrum to be compared to an observed spectrum. Two issues need to be addressed.

One has to determine the profile of each band to produce a full spectrum for every PAH considered. Then, one has to figure which families of PAHs are concerned, depending of the region of observation, and in which proportions they are to be averaged. 


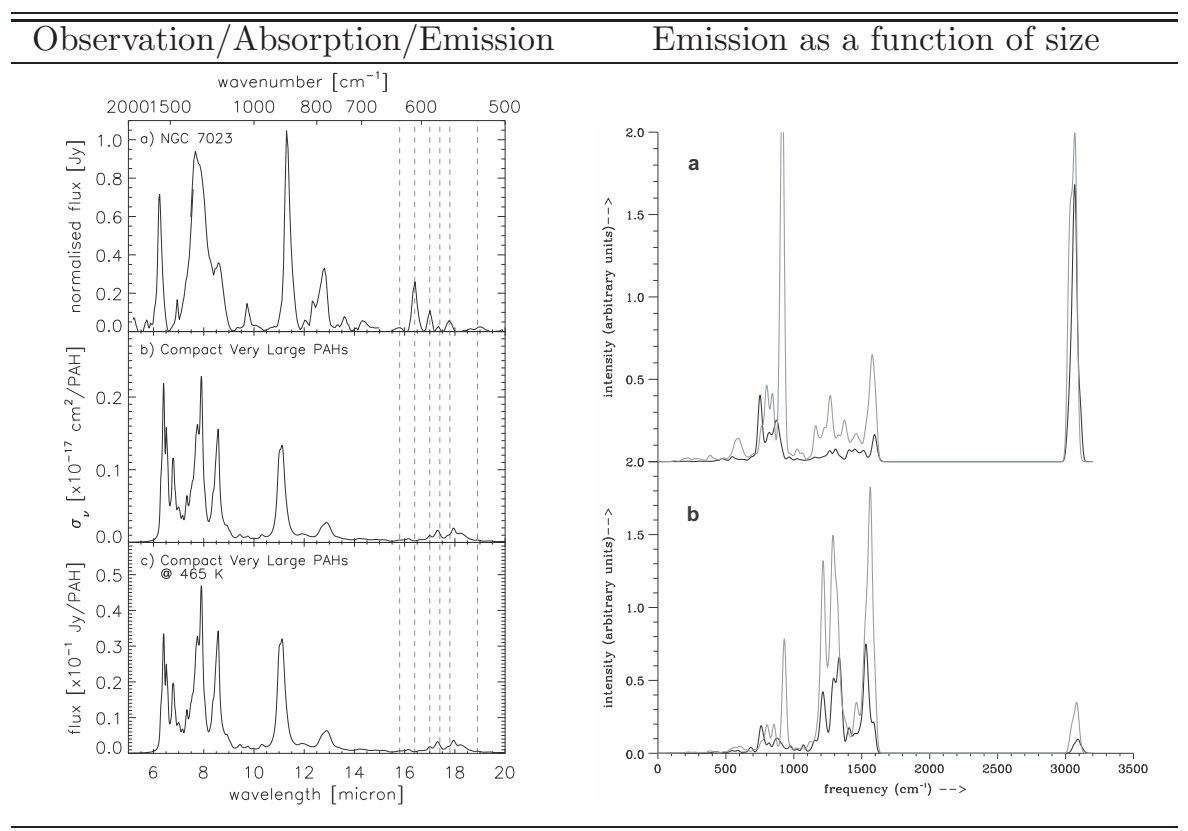

Fig. 3. Composite spectra: left - low-resolution spectrum of the reflection nebula NGC 7023 (panel a) compared to the average absorption spectrum of 7 neutral and singly positive ionised very large PAHs (panel b) and the computed emission spectrum of the same PAH mixture at $465 \mathrm{~K}$ (panel c) (adapted from Boersma 2009); right - comparative emission spectra of PAHs having less than $30 \mathrm{C}$ atoms (black) and more than $30 \mathrm{C}$ atoms (grey) (a) neutrals and (b) cations (adapted from Pathak \& Rastogi 2007).

In the case of a single PAH, it is now a priori possible to determine a theoretical profile for each band through elaborate methods implying intramolecular vibrational redistribution, anharmonic shifts and hot bands, rotational broadening, ... It is a highly sophisticated theoretical work, very demanding in man power and computer time. This is hardly an option to be considered when dealing with a mass production of spectra for such oversized molecules as PAHs. Empirical substitutes can be used instead.

Concerning the profile of a band, essentially two simplified representations are available:

- Histograms.

- Curves with Lorentzian / Gaussian shapes.

Then several options are possible for the $F W H M$ (full width at half maximum):

- The frequency interval obtained for the calculated vibrations of the same type 
- A standard line width

- Different FWHM depending on vibration type and deduced from experiments.

The final step is relevant of pure astrochemistry only. The appropriate calculated spectra have to be selected and averaged according variable percentages. Comparison of such composite spectra with the observed spectra allows to deduce information about size, ionization or composition of the PAHs according the environment. Some examples of composite spectra are given in Figure 3.

\section{Third generation computations: Current theoretical developments}

\subsection{Beyond electronic calculations: Anharmonicity}

To go beyond the harmonic approximation is not straightforward, but it is essential for understanding hot media in which the temperature influences the band profiles and the intensities as a consequence of the anharmonicity of the potential energy surfaces. Starting from a static quantum chemical calculation, Born Oppenheimer dynamics can be used at two levels of approximation:

- either the vibrations are considered as independent, which takes into account only one-dimension anharmonicity;

- or the vibrations are considered as coupled.

As for electronic calculations, variational or perturbative treatments can be employed, with similar pros and cons all linked to the size and nature of the basis set. A general option is to define a pool of vibrational states that can be populated at the temperature considered and reachable via allowed IR transitions.

The problem of size, in fact the dimension of the basis of vibrational functions so defined, becomes rapidly a bottleneck for the variational methods, especially in terms of computer time. For perturbation methods, it should be reminded that possible bias can be introduced by the perturbative technique used in most available codes, when accidental degeneracy occurs between vibrational levels, which is rather common when the size of the molecule and consequently the number of vibrations increases. The solution is then to resort to degenerate perturbation theory by solving variationally the manifold of quasi degenerate vibrational states in a preliminary step (see for example the case of naphthalene in Cané et al. 2007 or Pirali et al. 2009).

Using anharmonic parameters deduced from DFT results, it is possible to take the participation of every populated state to the overall profile of each IR band into account. But as the number of transitions and of states to be considered are quickly exploding with temperature, this type of calculation is so far limited to small PAHS. The description of really hot bands has to be done by using a statistical Monte Carlo sampling for example. (G. Mulas et al. 2006a; Pirali et al. 2009). 


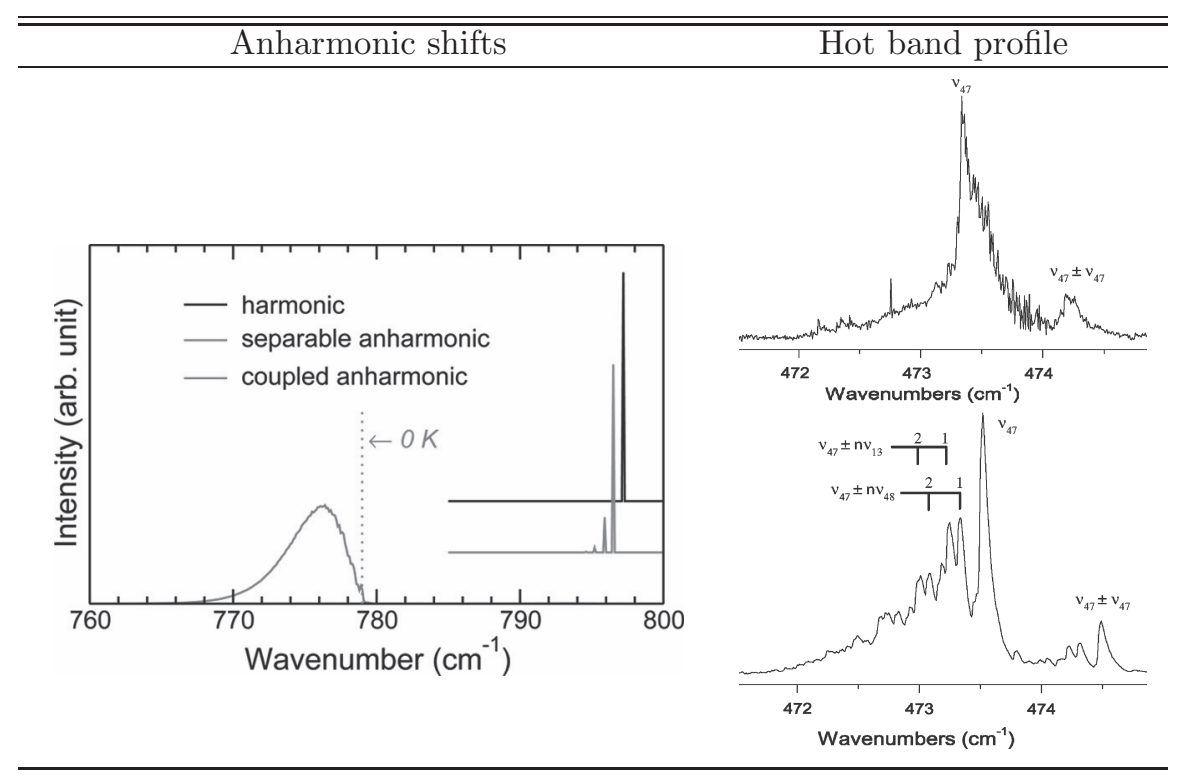

Fig. 4. Computational experiments on the absorption of naphthalene at fixed temperatures. Left: calculated partial spectrum near $780 \mathrm{~cm}^{-1}$ at $500 \mathrm{~K}$ (adapted from Basire et al. 2009); right: high resolution spectrum near $472 \mathrm{~cm}^{-1}$ at $300 \mathrm{~K}$ (adapted from Pirali et al. 2009); experimental (top); calculated (bottom).

Another theoretical approach is actually proposed to calculate the finite temperature IR absorption spectrum of fully coupled anharmonic systems. The energy levels are described by a second order Dunham expansion and a Wang Landau Monte Carlo procedure is used for the calculation of the quantum densities of states; microcanonical simulations provide an absorption intensity function of both the absorption wavelength and the internal energy of the molecule (Basire et al. 2008; Basire et al. 2009; see also Basire in this volume). Here too, applications are presently limited to the smallest of the PAHs.

Selected results from both methods are given in Figure 4.

For larger systems, anharmonic effects can be obtained by Born-Oppenheimer or Car-Parrinello molecular dynamics (MD). Though MD methods cannot provide as detailed structures as the preceding methods, it can give the overall band evolution with temperature, and has the advantage of being able to deal with much larger systems efficiently. For even more effectiveness, it can be coupled to computer efficient calculations of the potential energy function, as a parametrized DFT based on a tight binding approach such as SCC-DFTB (Self-Consistent Charge Density Functional Tight Binding (Porezag et al. 1995)); such an option used for SiPAH cations can be found in Joalland et al. (2010). 


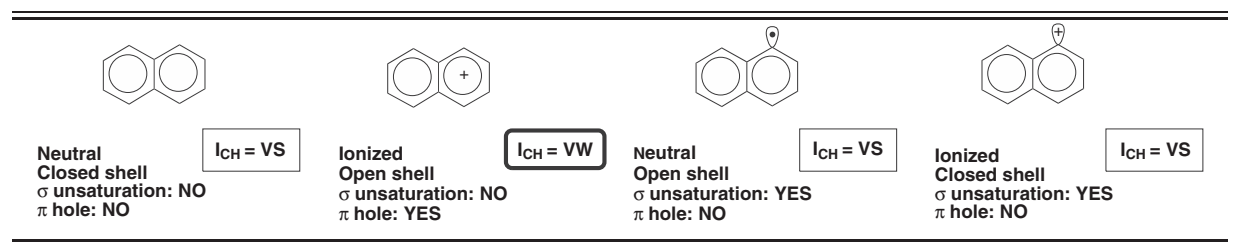

Fig. 5. Typical structures of neutral and ionized PAHs taken from the naphthalene series (adapted from Pauzat et al. 2010).

\subsection{Emission versus absorption spectra}

The a priori construction of emission spectra that is the last step toward a direct confrontation of theoretical models to the UIR bands has only been done recently. Two classes of emission models have been proposed.

The thermal emission model describes the IR cooling of PAHs via transitions from levels $\mathbf{v}$ to $\mathbf{v}-1$ in an emission cascade. The emission in a given vibrational mode from a PAH of internal energy $\mathbf{U}$ is calculated as the average emission of an oscillator connected to a thermal bath, the PAH itself being considered as a heat bath at the temperature $\mathbf{T}$ that corresponds to the average energy $\mathbf{E}$ of the molecule. This kind of model has been employed by Pech et al. (2002), using photophysical experimental data, as well as Pathak \& Rastogi (2008) using DFT B3LYP calculations $\left(\mathrm{C}_{10} \mathrm{H}_{8} \rightarrow \mathrm{C}_{96} \mathrm{H}_{24}\right)$ for the initial adsorption spectra. It has to be noted that this method is efficient but rather approximate: indeed, this canonical formalism is good only when the excitation energy is much larger than all the vibration modes considered, so that the average value of energy $\mathbf{E}$ is a sharply peaked function of $\mathrm{T}$; it is a poor approximation in all other cases.

Mulas and co chose a micro-canonical formalism. This approach is based on the conservation of energy between emissions, which insures its validity whatever the excitation energy considered. Though more difficult to implement than the thermal formalism, this option is more rigourous. Practically, the authors use a Monte Carlo technique on top of quantum chemical calculations by extending a preceding Monte Carlo model of photophysics to include rotational and anharmonic band structures and employing molecular parameters calculated by DFT (Mulas 1998; Mulas et al. 2006b).

\subsection{Origin of the collapse of the $\mathrm{CH}$ intensity with ionization}

Though the difference between the IR spectra of polycyclic aromatic hydrocarbons $(\mathrm{PAH})$ and those of the corresponding positive ions $\left(\mathrm{PAH}^{+}\right)$is nowadays a well admitted and documented fact, it remained puzzling and not clearly understood from a theoretical point of view till very recently. Interpreting the origin of these variations has long been neglected, because the production of IR spectra of all sorts of PAHs was considered a matter of urgency in order to support the observations and also because the tools available at the time were considered not reliable enough for such a delicate analysis. 


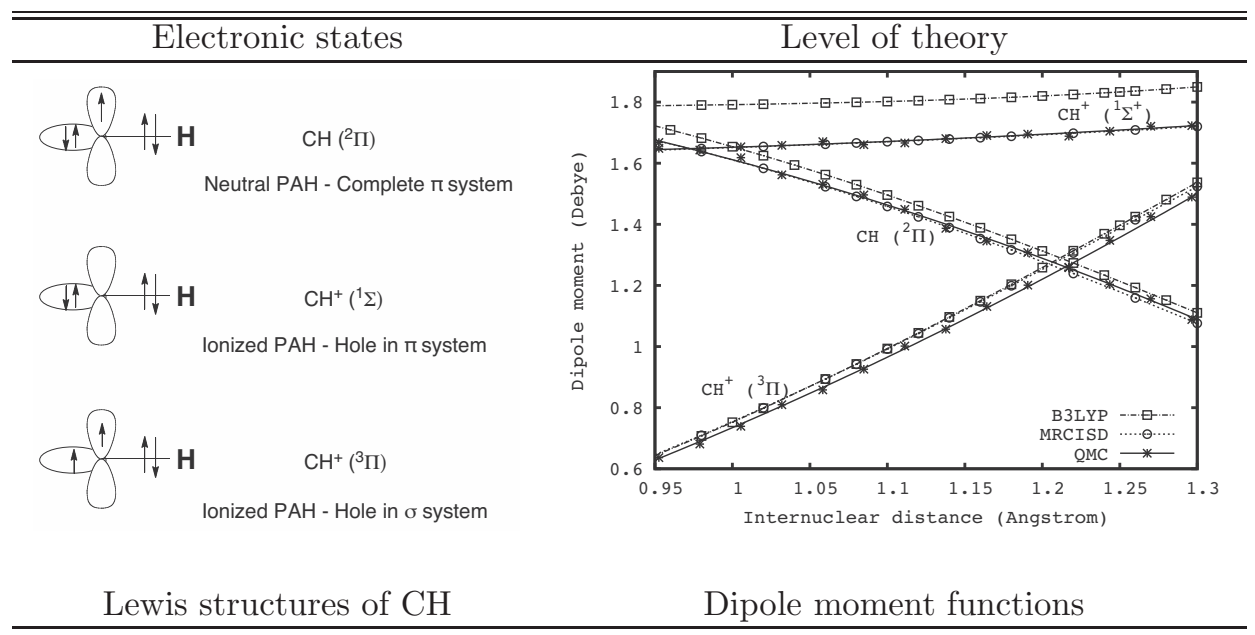

Fig. 6. Variation of the dipole moment of $\mathrm{CH}$ with the inter nuclear distance (adapted from Pauzat et al. 2010).

When looking more precisely to the large body of data available on PAHs, such a behavior seems to be linked to species with a hole in the system, which is the case of most classic ionized PAHs. A few demonstrative examples built from naphthalene are given hereafter in Figure 5 for a better understanding.

It is well known from vibrational analysis that the $\mathrm{CH}$ stretching vibration is a localized mode. Then, a local model of vibrator, namely, the $\mathrm{CH}$ fragment can represent the different types of PAHs as shown in Figure 6-left.

The study of the behavior of the dipole moment functions $\mu$ calculated with the most elaborate methods available, state of the art ab-initio (MRCISD) and Quantum Monte Carlo (QMC), showed that the three model molecules exhibit very different variations of their dipole moment with the $\mathrm{CH}$ distance (Fig. 6right) that are consistent with strong/weak intensities of the $\mathrm{CH}$ vibrations in the neutral/ionized PAHs, the key point being the presence, or not, of a hole in the $\pi$ shell. This can be interpreted as the result of the distorsions of the electronic densities, linked to different diabatic dissociation limits.

A topological analysis for the electronic densities (Pauzat et al. 2010) shows that the collapse of the $\mathrm{CH}$ stretching is directly linked to the compensation between the internal charge transfer contribution and the distortion of the electronic density within the $\mathrm{CH}$ bond, which occurs only in the case of $\mathrm{CH}^{+}\left({ }^{1} \Sigma^{+}\right)$.

\section{Conclusion}

The PAH hypothesis has been a real source of motivation for several theoretical groups over the last two decades. This is a quasi text book example of creative interaction between space observation, laboratory experiments and theoretical computations. The following summary of the marking theoretical contributions to the 


\begin{tabular}{|c|c|c|}
\hline Year & Landmarks & Research groups \\
\hline 1988 & $\begin{array}{l}\text { Poster IAU Symp. 135, Interstellar Dust } \\
\text { naphthalene; anthracene (neutral/cation) }\end{array}$ & $\begin{array}{c}\text { Miller/DeFrees } \\
\text { MRI-IBM }\end{array}$ \\
\hline $92-94$ & Effects of ionization $\left(\rightarrow \mathrm{C}_{16} \mathrm{H}_{10}\right)$ Obs. Paris/ENS & \\
\hline 93 & Anharmonicity and the $3.4 \mu \mathrm{m}$ band & $"$ \\
\hline 95-97 & Ionization versus dehydrogenation & $"$ \\
\hline 1996 & $\begin{array}{l}\text { Compilation of } 13 \text { PAHs (neutral/cation/anion) } \\
\rightarrow \mathrm{C}_{32} \mathrm{H}_{14} \text { (ovalene) }\end{array}$ & NASA Ames \\
\hline 97 & Perdeuterated small PAHs & $"$ \\
\hline 98 & Effect of $\mathrm{O} / \mathrm{N}$ substitution on Naphthalene & $"$ \\
\hline 99 & PAHs with 5-membered rings & $"$ \\
\hline \multirow[t]{3}{*}{99} & Effect of double ionization & Obs. Paris/ENS \\
\hline & Anthracene-Fe ${ }^{+}$complex & ., \\
\hline & Effect of aliphatic substitution $(3.4 \mu \mathrm{m}$ region $)$ & $"$ \\
\hline 2000 & $\begin{array}{l}\text { PAHs (neutral/cation/anion/ multiple cations) } \\
\rightarrow \mathrm{C}_{54} \mathrm{H}_{18} \text { (circumcoronene) }\end{array}$ & NASA Ames \\
\hline \multirow[t]{4}{*}{01} & Effects of perhydrogenation $(3.4 \mu \mathrm{m}$ region $)$ & Obs. Paris/ENS \\
\hline & Spectra of Perhydrogenated PAHs & NASA Ames \\
\hline & Large PAHs (neutral/protonated/cation) & \\
\hline & $\rightarrow \mathrm{C}_{96} \mathrm{H}_{24}$ (circumcircumcoronene) & \\
\hline \multirow[t]{3}{*}{02} & Effects of non-regularity (neutral/cation) & Obs. Paris/ENS \\
\hline & PAHs with $(4 / 5 / 7)$ membered rings & \\
\hline & Model for IR emission (band profiles) & U. Toulouse \\
\hline 03-05 & PANHs (neutral/cation) & NASA Ames \\
\hline 04 & Effect of deuteration & $"$ \\
\hline 05 & Catacondended PAHs ( (neutral/cation) & U. Gorakpur \\
\hline \multirow[t]{2}{*}{06} & Pericondended PAHs ( (neutral/cation) & \\
\hline & Far IR of PAHs: emission modeling & Obs. Cagliari \\
\hline \multirow[t]{2}{*}{07} & IR emission of PAHs dications & \\
\hline & $\mathrm{Fe}^{+}$PAH complexes & U. Toulouse \\
\hline 07-08 & PAH IR emission modeling and size effects & U. Gorakpur \\
\hline 08 & IR emission for PAHs with 10 to 96 carbons & \\
\hline \multirow[t]{4}{*}{09} & $\begin{array}{l}\text { IR absorption of thermally excited naphthalene } \\
\text { (anharmonic levels) }\end{array}$ & Obs. Cagliari \\
\hline & IR spectra of $\mathrm{Fe}^{+} / \mathrm{Mg}^{+} \mathrm{PAH}$ complexes & NASA Ames \\
\hline & Mid IR of very large irregular PAHs & \\
\hline & $\mathrm{C}_{84} \mathrm{H}_{24} \rightarrow \mathrm{C}_{120} \mathrm{H}_{36}$ & \\
\hline \multirow[t]{4}{*}{ 09-10 } & Anharmonic/temperature effects on IR spectra & U. Paris $X I$ \\
\hline & $\mathrm{SiPAH}^{+} \pi$ complexes (anharmonicity) & U. Toulouse \\
\hline & Far IR of large to very large PAHs $(\rightarrow 130 \mathrm{C})$ & NASA Ames \\
\hline & Origin of the collapse of the $3.3 \mu \mathrm{m}$ band & U. Paris $V I^{a}$ \\
\hline
\end{tabular}

a Formerly Obs. Paris/ENS.

Fig. 7. Chronology of the main advances in theoretical studies of PAH infrared spectra. 
knowledge of the infrared signatures of PAHs (Fig. 7) is certainly not exhaustive, the individual lonely contributions, except for the historical first one, not being quoted but only those of full groups. Some of these groups, among which the NASA Ames and Toulouse University/Cagliari groups have the particularity and the advantage to have an experimental group also dedicated to the PAH study, which allows them to constructive comparisons. These two groups are at the origin of the two data bases available. During the last years, new young research groups have entered the game and promoted real theoretical advances in particular for the problems of anharmonicity and emission.

The PAH story is still going on!

\section{References}

Allamandola, L.J., Tielens, A.G., \& Barker, J.R. 1985, ApJ, 290, L25

Amos, R.D., 1984, Chem. Phys. Lett., 108, 185

Bakes, E.L., Tielens, A.G., \& Bauschlicher, C.W., 2001, ApJ, 556, 501

Basire, M., Parneix, P., \& Calvo, F., 2008, J. Chem. Phys., 129, 081101

Basire, M., Parneix, P., Calvo, F., Pino, T., \& Bréchignac, Ph., 2009, J. Phys. Chem. A, 113, 6947

Bauschlicher, C.W., \& Langhoff, S.R., 1997, Spectrochim. Acta A, 53, 1225

Bauschlicher, C.W., Langhoff, S.R., Sandford, S.A., \& Hudgins, D.H., 1997, J. Phys. Chem. A, 101, 2414

Bauschlicher, C.W., Hudgins, D.M., \& Allamandola, L.J., 1999, Theor. Chem. Acc., 103, 154

Bauschlicher, C.W., \& Bakes, E.L., 2000, Chem. Phys., 262, 285

Bauschlicher, C.W., Hudgins, D.M., \& Allamandola, L.J., 2001, Theor. Chem. Acc., 105, 154

Bauschlicher, C.W., Peeters, E., \& Allamandola, L.J., 2008, ApJ, 678, 316

Bauschlicher, C.W., Peeters, E., \& Allamandola, L.J., 2009, ApJ, 697, 311

Bauschlicher, C.W., \& Ricca, A., 2009, ApJ, 698, 255

Bauschlicher, C.W., Boersma, C., Ricca, A., et al., 2010, ApJS, 189, 341

Becke, A.D., 1993, J. Chem. Phys., 98, 5648

Beegle, L.W., Wdowiak, T.J., \& Harrison, J.G., 2001, Spectrochim. Acta A, 57, 737

Boersma, C., 2009, PhD, U. Groeningen

Cané, E., Miani, A., \& Trombetti , A., 2007, J. Phys. Chem. A, 111, 8218

Cassam-Chenaï, P., Planet. Space Sci., 2002, 50, 871

DeFrees, D.J., Miller, M.D., Talbi, D., Pauzat, F., \& Ellinger, Y., 1993, ApJ, 408, 530

Duley, W.W., \& Williams, D.A., 1981, MNRAS, 196, 269

Ellinger, Y., Pauzat, F., \& Lengsfield, B. H., 1999, J. Mol. Struc., 458, 203

Helgaker, T.U., Jensen, H.J.A., \& Jorgensen, P., 1986, J. Chem. Phys., 84, 6280

Hudgins, D.H., Bauschlicher, C.W., \& Allamandola, L.J., 2005, ApJ, 632, 316

Joalland, B., Simon, A., Marsden, C. J., \& Joblin, C., 2009, A\&A, 494, 969

Joalland, B., Rapacioli, M., Simon, A., et al., 2010, J. Phys. Chem. A, 114, 5846 
Johnson, R.D., Irikura, K.K., Kacker, R.N., \& Kessel, R., 2010, J. Chem. Theory Comput., in press

Koch, W., \& Holthausen, M.C., 2001, A Chemist Guide to Density Functional Theory, 2nd ed. (Wiley-VCH, Weinheim, Germany)

Langhoff, S.R., 1996, J. Phys. Chem., 100, 2819

Langhoff, S.R., Bauschlicher, C.W., Hudgins, D.M., Sandford, S.A., \& Allamandola, L.J., 1998, J. Phys. Chem. A, 102, 1632

Lee, C., Yang, W., \& Parr, R.G., 1988, Phys. Rev. B, 37, 785

Lee, T.J., \& Scuseria, G.E., 1995, In "Quantum mechanical electronic structure calculations with chemical accuracy", ed. S.R. Langhoff (Kluwer Academic Publishers: Dordrecht, The Netherlands)

Léger, A., \& Puget, J.L., 1984, A\&A, 137, L5

McLean, A.D., Lengsfield, B.H., Pacansky, J., \& Ellinger, Y., 1985, J. Chem. Phys., 83, 3567

Malloci, G., Joblin, C., \& Mulas, G., 2007, Chem. Phys., 332, 353

Malloci, G., Joblin, C., \& Mulas, G., 2007, A\&A, 462, 627

Martin, J.M.L., El-Yazal, J., \& François, J.P., 1994, J. Phys. Chem., 98, 4243

Merrick, J.P., Moran, D., \& Radom, L., 2007, J. Phys. Chem. A, 111, 11683

Miller, M.D., \& DeFrees, D.J., 1988, IAU Symp. 135, Interstellar Dust, Santa Clara

Mulas, G., 1998, A\&A, 338, 243

Mulas, G., Malloci, G., \& Benvenuti, P., 2003, A\&A, 410, 639

Mulas, G., Malloci, G., Joblin, C., \& Toublanc, D., 2006a, A\&A, 456, 161

Mulas, G., Malloci, G., Joblin, C., \& Toublanc, D., 2006b, A\&A, 460, 93

Pathak, A., \& Rastogi, S., 2007, Advances in Space Research, 40, 1620

Pathak, A., \& Rastogi, S., 2007, Spectrochim. Acta Part A, 67, 898

Pathak, A., \& Rastogi, S., 2008, A\&A, 485, 735

Pauzat, F., Talbi, D., Miller, M.D., DeFrees, D.J., \& Ellinger, Y., 1992, J. Phys. Chem., 96,7882

Pauzat, F., Talbi, \& Ellinger, Y., 1995, A\&A, 293, 263

Pauzat, F., Talbi, \& Ellinger, Y., 1997, A\&A, 319, 318

Pauzat, F., Talbi, \& Ellinger, Y., 1999, MNRAS, 304, 241

Pauzat, F., \& Ellinger, Y., 2001, MNRAS, 324, 355

Pauzat, F., \& Ellinger, Y., 2002, Chem. Phys., 280, 267

Pauzat, F., Pilmé, J., Toulouse, J., \& Ellinger, Y., 2010, J. Chem. Phys., 133, 054301

Pech, C., Joblin, C., \& Boissel, P., 2002, A\&A, 388, 639

Peeters, E., Allamandola, L.J., Bauschlicher, C.W., et al., 2004, ApJ, 604, 252

Pirali, O., Vervloet, M., Mulas, G., Malloci, G., \& Joblin, C., 2009, Phys. Chem. Chem. Phys., 11, 3443

Porezag, D., Frauenheim, T., Köhler, T., Seifert, G., \& Kaschner, R., 1995, Phys. Rev. B, 51, 12947

Ricca, A., Bauschlicher, C.W., Mattioda, A.L., Boersma, C., \& Allamandola, L.J., 2010, ApJ, 709, 42

Ricks, A.M., Douberly, G.E., \& Duncan, M.A., 2009, ApJ, 702, 301 
Scott, A., \& Radom, L., 1996, J. Phys. Chem. A, 100, 16502

Simon, A., \& Joblin, C., 2007, J. Phys. Chem., 111, 9745

Simon, A., \& Joblin, C., 2010, ApJ, 712, 69

Szabo, A., \& Ostlund, N.S., 1982, Modern Quantum Chemistry (McGraw-Hill, New York)

Wakelam, V., \& Herbst, E., 2008, ApJ, 680, 371

Yoshida, H., Ehara, A., \& Matsuura, H., 2000, Chem. Phys. Lett., 325, 477 
